\title{
Role of laparoscopy in diagnosis of peritoneal tuberculosis (About 414 cases)
}

\author{
H. Abid, I. Charif, I. Mellouki, N. Aqodad, D. Benajah, A. Ibrahimi, M. El Abkari, \\ M. El Yousfi
}

Department of Hepatology and Gastroenterology, Hassan II University Hospital of Fez, Fez, Morroco

Email: abidhakima@hotmail.com

Received 29 April 2013; revised 30 May 2013; accepted 10 June 2013

Copyright (C) 2013 H. Abid et al. This is an open access article distributed under the Creative Commons Attribution License, which permits unrestricted use, distribution, and reproduction in any medium, provided the original work is properly cited.

\begin{abstract}
Peritoneal tuberculosis is still common in Morocco. Its diagnosis is based on several methods. Laparoscopy with directed biopsies is still considered as the referenced method. The aim of our work is to show the place and the importance of laparoscopy in the diagnosis of peritoneal tuberculosis through the study of various endoscopic aspects. Materials and Methods: This is a retrospective descriptive study extended from January 2001 to December 2011. We collected 414 cases of isolated ascites confirmed by ultrasonography. Different parameters were analyzed: epidemiological data, indications of laparoscopy and its results and complications. Results: The average age of our patients was 38 years (range: 8 years - 90 years) with a female predominance (sex ratio F/M: 2.3). Abdominal ultrasound performed in all patients has confirmed the presence of ascites in $100 \%$ of cases. Based on clinical data (soft or tense abdomen) and ultrasound (free or partitioned ascites), we performed a standard laparoscopy in 313 cases and open laparoscopy in 101 cases. The presence of granules was noted in 318 cases $(76.8 \%)$. Peritoneal tuberculosis was found in $\mathbf{8 1 . 5 \%}$ of cases with small whitish granulations distributed homogeneously, while peritoneal carcinomatosis was confirmed in $86.8 \%$ of patients with large granules distributed inhomogeneously. The presence of adhesions was observed in $33 \%$ of cases with peritoneal tuberculosis, while it was mentioned in only 2.8 cases of carcinomatosis. Conclusion: Despite the development of imaging means, ascitic fluid culture and PCR (polymerase chain reaction), laparoscopy remains indispensable for an early diagnosis of peritoneal tuberculosis and adequate management.
\end{abstract}

Keywords: Peritoneal Tuberculosis; Laparoscopy

\section{INTRODUCTION}

Peritoneal tuberculosis is a public health problem, especially in the third world countries. It is responsible for high morbidity and mortality. Peritoneal tuberculosis accounts for $5 \%$ of all locations. The association with pulmonary tuberculosis has been reported in $20 \%$ to $50 \%$ of cases [1,2]. Peritoneal tuberculosis may originate from a lymphatic (mesenteric lymph nodes) or through blood or be secondary to tuberculous salpingitis [3]. Clinical symptoms may be insidious with abdominal pain, sweating, anorexia, fever and weight loss. Laparoscopy with biopsy remains the gold standard for the diagnosis. Our work highlights the importance of laparoscopy as an indispensable mean for the diagnosis of peritoneal tuberculosis which remains common in our country.

\section{MATERIELS AND METHODS}

This is a retrospective descriptive study extended from January 2001 to December 2011, in the gastroenterology unit of the Hassan II university hospital of Fez. 414 diagnostic laparoscopies were performed during this period. Different parameters are analyzed: epidemiological data, indications, results of laparoscopy and its complications.

\section{RESULTS}

During the study period of 10 years, 414 laparoscopies were done. Peritoneal tuberculosis was confirmed histologically in 294 cases (71\%), while peritoneal carcinomatosis was found in 68 cases (16.4\%). Histology was inconclusive in 45 cases (10\%).

Among the 294 cases of peritoneal tuberculosis collected, the average age of our patients was 38 years (range: 8 years - 90 years), with a marked female predominance (sex ratio F/M: 2.3). The majority of patients were of low socioeconomic level. The clinical symptomatology was dominated by an increase in abdominal 
volume and abdominal pain. Abdominal ultrasound performed in all our patients had confirmed the presence of ascites in $100 \%$ of cases. Laboratory tests revealed an inflammatory syndrome with an elevated erythrocyte sedimentation rate in $75 \%$ of cases. The tuberculin skin test was rarely positive. A study of ascites fluid was performed and objectified lymphocytic exudate in all our patients.

Based on data from the clinical examination (soft or tense abdomen) and ultrasound (freeor partitioned ascites) we performed a standard laparoscopy in 227 cases and open laparoscopy in 67 cases. Endoscopically, we analyzed the following data:

- The appearance of the peritoneum

- The granules: size, location, color, distribution

- The adhesions

- Appearance of the liver

Thus, we noted the presence of granules in $78 \%$ of cases of tuberculosis. In $64 \%$ of cases, these granules were small, white andhomogeneously distributed on the peritoneum. In cases of carcinomatosis, granulations were large and inhomogeneously distributed in $86.8 \%$ of cases (Figure 1). Adhesions were observed in 52\% of cases of peritoneal tuberculosis while they were found in only 2.8 of cases of carcinomatosis. The liver was seen in 167 cases and was normal in $53 \%$ of cases.

Complications of laparoscopy were observed in 10 cases (2.4\% of cases) including 8 cases of perforation, one case of air embolism and one case of vagal shock.

\section{DISCUSSION}

The location of peritoneal tuberculosis remains a frequent localization in highly endemic countries such as Morocco. The situation is widely different in developed countries where peritoneal tuberculosis is a rare localization. It currently represents $0.5 \%$ of new peritoneal tuberculosis cases and $3.8 \%$ of extra-pulmonary tuberculosis in the United States and 3.7\% in France [4]. It is the

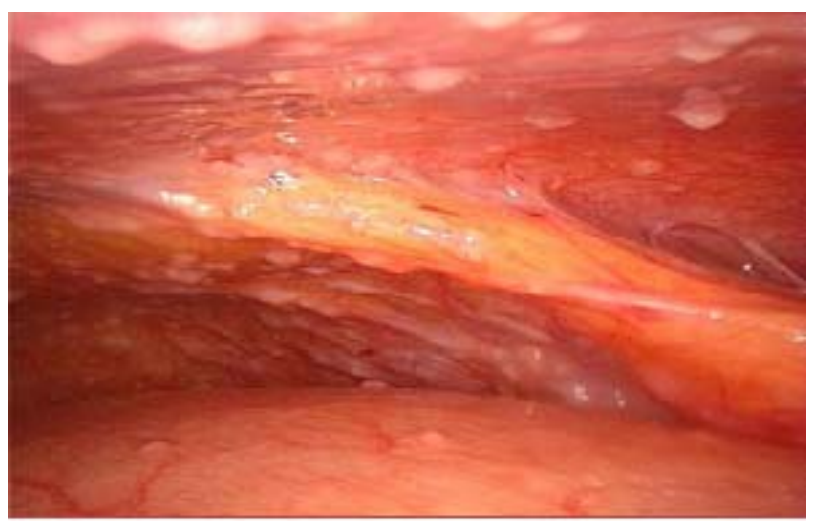

Figure 1. Laparoscopy image objectifying the presence of granulations and peritoneal adhesions. most common form of abdominal locations [3]. Symptomatology is dominated by abdominal pain associated with abdominal distension related to free or partitioned ascites. More insidious symptoms can be noticed with asthenia, anorexia, weight loss and fever. Laboratory tests may show a nonspecific inflammatory syndrome with increased erythrocyte sedimentation rate, C-reactive protein. The intradermal tuberculin reaction is often negative.

Detection of the organism (Mycobacterium tuberculosis) in the ascites fluid is very difficult and it is positive on direct examination in $5 \%$ of cases $[5,6]$. The seed culture on specific environment allows a definitive diagnosis, but requires a long time to get the results. The molecular diagnostic methods are of great interest, but are often not available especially in developing countries. In our series, no PCR was performed.

Histology is the appropriate method for the diagnosis of peritoneal tuberculosis and the diagnosis of other diseases such as peritoneal carcinomatosis.

Laparotomy was once the only way to confirm the diagnosis of peritoneal tuberculosis. Currently laparoscopy is the most effective abdominal exploration in suspected cases of peritoneal tuberculosis. Indeed, it allows establishing a rapid diagnosis and showing some specific lesions (peritoneal granulations, adhesion, and inflammatory aspect of the peritoneum) and allows peritoneal biopsies. Laparoscopy allows the diagnosis of peritoneal tuberculosis in $72 \%-97 \%$ of cases [7]. But this technique haslimitations and complications are minimal compared to the laparotomy. They are of the order of $3 \%$ according to the literature [8]. These complications are mainly represented by hemorrhage and intestinal perforation (in our series, 8cases of perforation (2\%) were observed). To minimize these risks some authors [7] prefer the "open laparoscopy" (in our series, of a total of 414 laparoscopies performed, 101 cases underwent open laparoscopies.

\section{CONCLUSION}

Our work contributes to demonstrate the important role of laparoscopy in the diagnosis of peritoneal tuberculosis, allowing to study the endoscopic features and implement biopsies for histological confirmation. This technique is more efficient with a higher yield than other means of diagnosis with minimal complications.

\section{REFERENCES}

[1] Marshall, J.B. (1993) Tuberculosis of gastrointestinal tract and peritoneum. American Journal of Gastroenterology, 88, 989-999.

[2] Chem, Y., Lee, D.Y. and Perng, P.R. (1955) Abdominal tuberculosis in Taiwan a report from veterans, general hospital, Taipei. Tuber Lung Disease, 76, 35-38. 
[3] Zafarpore, F., et al. (2007) The role of laparoscopy in the diagnosis of abdominal tuberculosis. The Saoudi Journal of Gastroenterology, 13, 133-135.

[4] Fox, H., Ollivier, C., Le Gmann, P. and Levesque, M. (1987) Tuberculose péritonéale à propos d'une observation. Annales De Pediatrie (Paris), 34, 735-738.

[5] Bhargava, D.K. (1992) Peritoneal tuberculosis: Laparoscopic patterns and its diagnostic accuracy. American Journal of Gastroenterology, 87, 109-112.

[6] Talavera, W. and Lessnau, K.D. (1994) Extrapulmonary tuberculosis in fiedman in tuberculosis current concepts and treatment. Ann. Arbor. London, 113-151.

[7] Sandicki, M., Colakoglus, S., Ergun, Y., et al. (1992) Présentation and role of peritoneoscopy in the diagnosis of tuberculosis. Journal of Gastroenterology and Hepatology, 7, 298-301. doi:10.1111/j.1440-1746.1992.tb00984.x

[8] Mansoor, A. and Aijaz, R. (1992) Tuberculosis peritonitis; fatality associated with delayed diagnosis. Southern $\mathrm{Me}$ dical Journal, 92, 406-440. 